\section{Comments on Zinc Formalin Fixative}

Richard W. Dapson, Anatech Ltd. anatech@net-link.net

Questions have arisen recently on the Histonet listserver about the properties of zinc formalin fixative. The advantage of zinc formalin over the usual formalin fixatives appears to be that zinc ions hold macromolecules in their native conformation via coordinate bonds, preventing the damaging crosslinkages that formaldehyde alone would create. The result is greatly enhanced immunopreservation: rarely is antigen retrieval necessary, and primary antibodies can be diluted 2 to 10 fold greater than usual.

The original use of zinc formalin (by $P$. Banks and coworkers) was to prevent nuclear bubbling artifact in tissues fixed for less than 24 to 48 hours. Because nuclear morphology is so sharply defined, zinc formalin is frequently used as a replacement for B-5 mercuric fixative.

Today there are several distinct varieties of zinc formalin, but all share the advantages mentioned above. The original formula for zinc formalin is $1 \%$ zinc sulfate heptahydrate in $10 \%$ unbuffered formalin. It tends to precipitate in tissue processors, as the zinc is not soluble in the $70 \%$ alcohol used in the first dehydration station. This can cause lines to plug if routine acidic rinses are not performed. Neutral or alkaline water will not dissolve the precipitate. Some processor manufacturers do not want zinc formalin in their machines because of this. The zinc inside the specimens also precipitates (it cannot be seen), and causes difficulties in microtomy.

Anatech Ltd. developed an unbuffered zinc sulfate formula that does not precipitate in $70 \%$ alcohol (it will precipitate if you go directly from this formula to $80 \%$ or higher alcohol), and can be used in all processors. Tissues are not crunchy, and in fact customers often remark that they are easier to cut than those fixed in neutral buffered formalin.

All unbuffered zinc formalins are acidic $(\mathrm{pH}$ varies from about 3 up to about 4.5). Formalin pigment will develop below about $\mathrm{pH} 5.3$, faster as $\mathrm{pH}$ gets lower. The vast majority of our customers using unbuffered zinc formalin do not report formalin pigment artifact (which frankly surprised us), and I can only assume that it is because exposure time is too short for it to happen. Given comparable $\mathrm{pH}$ levels, acidic zinc formalin and acidic (unbuffered) formalin will behave similarly regarding pigment formation.

Buffered zinc formalin is also available, but again, some formulations precipitate badly in processors. Anatech's product (Z-Fix) is freely soluble in alcohol, and can be made as an alcoholic buffered zinc formalin for those of you who like the added benefits (speed, penetration of fat) that alcoholic formalin provides:

Most zinc formalin solutions do not corrode metal any faster than formaldehyde will. Remember that formaldehyde is rather corrosive to nearly all steel, including most forms of stainless steel (316 and 410 stainless are safe).

One group of zinc formalin solutions, those made with zinc chloride, is highly corrosive. Faster than its cousins and nearly as aggressive as mercuric chloride, zinc chloride has been used (not by us) as a B-5 replacement. It can overfix tissues, just like B-5 does, and absolutely must not be put in an enclosed processor. There are very effective rapid, zinc-based B5 replacements that do not contain the chloride salt.

Anatech's products are available only in the US and Can- ada. Shandon Lipshaw has a processor-compatible zinc formalin (unbuffered) that is available worldwide. I believe that RichardAllan's zinc formalin product line is available only in the U.S. A comprehensive review of zinc formalin up to 1992 appears in the following paper: R.W. Dapson, 1993. Fixation for the 1990 's: a review of needs and accomplishments. Biotechnic \& Histochem 68:75-82.

\section{A Low-Cost Salt Plate Holder for Micro FT-IR \\ James Benko, Microspec Analytical \\ jim.benko@mspec.com}

FT-IR (Fourier Transform Infrared) spectroscopy is an instrumental technique for measuring the infrared spectrum of materials. Many organic compounds have characteristic spectra that can be used for their identification, and specific functional groups (hydroxyl, carbonyl. amine, etc.) have characteristic bands at certain frequencies.

Microscopes have been coupled to FT-IR instruments and allow the IR spectrum determination of single particles. This greatly increases the identification possibilities of small particles and contaminants. These particles can be mounted on sodium chloride or potassium bromide salt crystals, since these materials are transparent to infrared radiation. Round, highly polished crystals can be purchased that fit in specific holders supplied by the instrument manufacturers, but often a small piece of crystal taken from a larger, discarded or broken salt plate used for regular infrared work can be used effectively. This provides significant cost savings, and when used in the easily constructed holder described below, the entire sample holder is disposable.

This low cost holder for micro salt plates can be made from cardboard and poster mounting squares.

1) Cut the cardboard with scissors to fit the conventional holder for

micro salt plates as required.

2) Punch out holes where desired using a $1 / 4$ " diameter hole punch.

3) Make similar holes through a poster mounting square (such as Scotch No.111 one inch poster mounting squares or equivalent). This can be done with a small soldering iron or a small cork borer.

4) Remove the protective paper and put the squares over the holes made in the cardboard holder. The bottom surface of each poster square is sufficient to hold it to the cardboard holder. The top sticky surface serves to hold scrap pieces of salt crystals for micro FT-IR work.

As an alternative to using cardboard, the poster squares can be put directly onto original equipment manufacturers' salt plate holders. Instead of using the small cut circles of salt that fit these holders, any random cut or scraps from broken salt plates can be used.

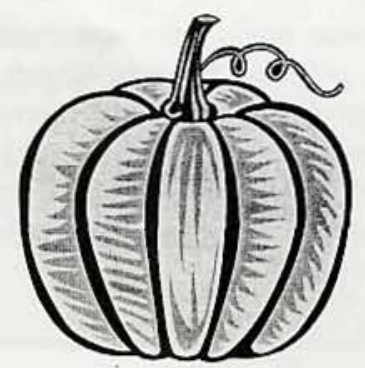

